\title{
Rotating D3-branes and QCD in three dimensions
}

\author{
Jorge G. Russo ${ }^{a}$, Konstadinos Sfetsos $^{b}$ \\ ¿Departamento de Física, \\ Universidad de Buenos Aires, \\ Ciudad Universitaria, \\ 1428 Buenos Aires, Argentina \\ russo@df.uba.ar \\ ${ }^{b}$ Theory Division, \\ CERN \\ CH-1211 Geneva 23, \\ Switzerland \\ sfetsos@mail.cern.ch
}

\begin{abstract}
We investigate the rotating D3-brane solution with maximum number of angular momentum parameters. After determining the angular velocities, Hawking temperature, ADM mass and entropy, we use this geometry to construct general three-parameter models of nonsupersymmetric pure $S U(N)$ Yang-Mills theories in 2+1 dimensions. We calculate glueball masses in the WKB approximation and obtain closed analytic expressions for generic values of the parameters. We also determine the masses of Kaluza-Klein states associated with internal parts of the ten-dimensional metric and investigate the parameter region where some of these states are decoupled. To leading order in $1 / \lambda$ and $1 / N$ (where $\lambda$ is the 't Hooft coupling) we find a global $U(1)^{3}$ symmetry and states with masses comparable to glueball masses, which have no counterpart in the more familiar (finite $\lambda, N$ ) Yang-Mills theories.
\end{abstract}




\section{Introduction}

The extremal D3-brane provides one of the simplest illustrations of the recently found dualities between gauge theories and string theory on geometries which asymptotically approach the Anti-de Sitter space-time [1]. Because the string models on these geometries are not understood to date, much of the attention has been devoted to those models where the curvature of the geometry is small everywhere, so that the system can be studied by using supergravity. This is the case for black brane geometries, which for large charges have small curvatures everywhere outside the horizon. The most general geometry with a regular horizon, which has a D3-brane charge, is given by the non-extremal D3-brane with three angular momenta. It is of obvious interest to investigate the spectra of this general (five-parameter) supergravity model based on the D3-brane. In this paper we determine the spectrum of the corresponding Laplace operator in the WKB approximation.

Models of QCD in $2+1$ dimensions can be constructed from the nonextremal D3-brane geometry by compactifying the euclidean time direction, which plays the role of an internal angle [2-7]. In the case of zero angular momentum, there is a single mass scale in the spectrum (given by the Hawking temperature); Kaluza-Klein states associated with the compact Euclidean time have the same mass scale as the states with vanishing Kaluza-Klein charge (e.g. glueballs) and the dimensional reduction is not justified. This problem can be overcome by starting with a rotating D3-brane with large angular-momentum, in which case the radius of the circle shrinks to zero and the Kaluza-Klein particles decouple [5, 7]. Another problem in making contact with ordinary Yang-Mills theory was pointed out in [8]. While the static D3-brane has an $S O(6)$ isometry group associated with the fivesphere part of the geometry, in pure non-supersymmetric Yang-Mills theory there is no counterpart of this $S O(6)$ global symmetry. Introducing angular momentum breaks the $S O(6)$ isometry group to smaller subgroups. When the maximum number of angular momentum components are turned on, the only remaining global symmetry is the Abelian (Cartan) subgroup $U(1) \times U(1) \times U(1)$ of $S O(6)$. Although this is in a sense closer to QCD than a model based on the static D3-brane (which implies a large $S O(6)$ global symmetry), there is no Abelian $U(1)^{3}$ global symmetry in weakly coupled non-supersymmetric $S U(N)$ Yang-Mills theory without matter. A natural question is whether the Kaluza-Klein particles, which are charged with respect to the $U(1)^{3}$ Cartan group, could have large masses (with respect to the glueball masses) in some region of the three-dimensional parameter space $a_{1}, a_{2}, a_{3} .{ }^{1}$ In Sect. 4.3 it will be shown that in the supergravity approximation these Kaluza-Klein particles cannot decouple in any region of

\footnotetext{
${ }^{1}$ There are two extra parameters associated with charge and mass of the D3-brane: the mass fixes the scale and can be set to 1 ; the charge is related to the 't Hooft coupling $\lambda$ and in the supergravity approximation does not affect the mass spectra.
} 
the parameter space $a_{1}, a_{2}, a_{3}$. It should be noted that the WKB approximation is sufficient to establish that certain states do not decouple, since one can consider in particular states with sufficiently high radial quantum numbers so that the WKB approximation is close to the exact result. It is nevertheless reasonable to expect that these exotic Kaluza-Klein particles may decouple once all $1 / \lambda$ effects are incorporated, since the weakly coupled non-supersymmetric Yang-Mills theory that should govern the low-energy dynamics of the compactified D3-brane does not have these particles in the physical spectrum. A further discussion on this is given in Sect. 5 .

\section{The rotating D3-brane background}

The D3-brane has an internal $S O(6)$ rotational isometry, which allows three independent angular parameters, $l_{1}, l_{2}, l_{3}$. The metric for the case $l_{2}=l_{3}=0$ was given in [5]. The general metric with parameters $l_{1}, l_{2}, l_{3}$ was recently given in an appendix in [9] and was obtained by duality transformations of the black hole solutions of [10] (there are two typographical errors in the expression of [9] that are corrected below). The rotating D3-brane metric is given by

$$
\begin{aligned}
d s_{\mathrm{IIB}}^{2} & =f_{0}^{-1 / 2}\left(-h_{0} d x_{0}^{2}+d x_{1}^{2}+d x_{2}^{2}+d x_{3}^{2}\right)+f_{0}^{1 / 2}\left[\frac{\Delta d r^{2}}{\prod_{i=1}^{3}\left(1+\frac{l_{i}^{2}}{r^{2}}\right)-\frac{2 m}{r^{4}}}\right. \\
& +r^{2}\left(\Delta_{1} d \theta^{2}+\Delta_{2} \cos ^{2} \theta d \psi^{2}-2 \frac{l_{2}^{2}-l_{3}^{2}}{r^{2}} \cos \theta \sin \theta \cos \psi \sin \psi d \theta d \psi\right. \\
& +\left(1+\frac{l_{1}^{2}}{r^{2}}\right) \sin ^{2} \theta d \varphi_{1}^{2}+\left(1+\frac{l_{2}^{2}}{r^{2}}\right) \cos ^{2} \theta \sin ^{2} \psi d \varphi_{2}^{2} \\
& +\left(1+\frac{l_{3}^{2}}{r^{2}}\right) \cos ^{2} \theta \cos ^{2} \psi d \varphi_{3}^{2}+\frac{2 m}{r^{6} \Delta f_{0}}\left(l_{1} \sin ^{2} \theta d \varphi_{1}\right. \\
& \left.\left.+l_{2} \cos ^{2} \theta \sin ^{2} \psi d \varphi_{2}+l_{3} \cos ^{2} \theta \cos ^{2} \psi d \varphi_{3}\right)^{2}\right)-\frac{4 m \cosh \alpha}{r^{4} \Delta f_{0}} d x_{0} \\
& \left.\times\left(l_{1} \sin ^{2} \theta d \varphi_{1}+l_{2} \cos ^{2} \theta \sin ^{2} \psi d \varphi_{2}+l_{3} \cos ^{2} \theta \cos ^{2} \psi d \varphi_{3}\right)\right]
\end{aligned}
$$

where

$$
\begin{aligned}
\Delta & =1+\frac{l_{1}^{2}}{r^{2}} \cos ^{2} \theta+\frac{l_{2}^{2}}{r^{2}}\left(\sin ^{2} \theta \sin ^{2} \psi+\cos ^{2} \psi\right)+\frac{l_{3}^{2}}{r^{2}}\left(\sin ^{2} \theta \cos ^{2} \psi+\sin ^{2} \psi\right) \\
& +\frac{l_{2}^{2} l_{3}^{2}}{r^{4}} \sin ^{2} \theta+\frac{l_{1}^{2} l_{3}^{2}}{r^{4}} \cos ^{2} \theta \sin ^{2} \psi+\frac{l_{1}^{2} l_{2}^{2}}{r^{4}} \cos ^{2} \theta \cos ^{2} \psi \\
\Delta_{1} & =1+\frac{l_{1}^{2}}{r^{2}} \cos ^{2} \theta+\frac{l_{2}^{2}}{r^{2}} \sin ^{2} \theta \sin ^{2} \psi+\frac{l_{3}^{2}}{r^{2}} \sin ^{2} \theta \cos ^{2} \psi \\
\Delta_{2} & =1+\frac{l_{2}^{2}}{r^{2}} \cos ^{2} \psi+\frac{l_{3}^{2}}{r^{2}} \sin ^{2} \psi \\
h_{0} & =1-\frac{2 m}{r^{4} \Delta}, \quad f_{0}=1+\frac{2 m \sinh ^{2} \alpha}{r^{4} \Delta} .
\end{aligned}
$$


The dilaton field $\Phi$ is constant, $e^{\Phi}=g_{s}$. The angular part differs from eq. (69) of [9] in the components $G_{\theta \theta}$ and $G_{\psi \psi}$. Some interesting thermodynamical aspects of rotating D3-branes have recently been investigated in refs. $[11,9,12]$ in the context of gauge/string-theory correspondence, which indicate that there may be a phase transition in $\mathcal{N}=4$ super-Yang-Mills theory at finite temperature $[11,12]$. At zero temperature the supergravity solution corresponds to the continuum limit $[9,13]$ of a multicenter static D3-brane solution and describes a Higgs phase of $\mathcal{N}=4$ super-Yang-Mills theory with a vacuum having a $Z_{N}$-type symmetry [13]. It would be interesting to extend these discussions to the general case (1) of maximum number of angular momenta. The interpretation is somewhat different in the present case, where (1) will be used to construct a static space-time, with the Euclidean time parametrizing an internal circle.

The parameters $m$ and $\alpha$ are related to the D3-brane charge $N$ by

$$
\sinh ^{2} \alpha=\sqrt{\left(2 \pi g_{s} N \alpha^{\prime 2} / m\right)^{2}+1 / 4}-1 / 2 .
$$

For completeness we also include the 4-form gauge field [9]

$$
\begin{aligned}
C^{(4)}= & \frac{1-f_{0}^{-1}}{\sinh \alpha} d x_{1} \wedge d x_{2} \wedge d x_{3} \wedge\left(\cosh \alpha d t-l_{1} \sin ^{2} \theta d \varphi_{1}\right. \\
& \left.-l_{2} \cos ^{2} \theta \sin ^{2} \psi d \varphi_{1}-l_{3} \cos ^{2} \theta \cos ^{2} \psi d \varphi_{1}\right) .
\end{aligned}
$$

The location of the horizon $r=r_{H}$ is given by the largest real root of

$$
\prod_{i=1}^{3}\left(r^{2}+l_{i}^{2}\right)-2 m r^{2}=0
$$

which is a cubic equation for $r^{2}$. The angular velocities $\Omega_{1}, \Omega_{2}, \Omega_{3}$ associated with motion in $\varphi_{1}, \varphi_{2}, \varphi_{3}$ can be determined by requiring that the vector $\eta=\frac{\partial}{\partial x_{0}}+\Omega_{i} \frac{\partial}{\partial \varphi_{i}}$ be null at the horizon. They are independent of the angles, so that one can compute them by evaluating $\eta^{2}$ at different values of $\theta$ and $\psi$. At $\theta=0, \psi=0, \eta^{2}$ is independent of $\Omega_{1,2}$, so that setting $\eta^{2}=0$ determines $\Omega_{3}$. Similarly, by evaluating $\eta^{2}$ at $\theta=0, \psi=\pi / 2$, and using the value of $\Omega_{3}$ already obtained, one finds $\Omega_{2}$, whereas by evaluating it at $\theta=\pi / 2, \psi=\pi / 2$ one obtains $\Omega_{1}$. The result can be written compactly as

$$
\Omega_{i}=\frac{l_{i}}{\cosh \alpha\left(r_{H}^{2}+l_{i}^{2}\right)}, \quad i=1,2,3 .
$$

The Hawking temperature is obtained from $\eta^{2}$ by the formula

$$
T_{H}^{2}=\lim _{r \rightarrow r_{H}} \frac{1}{16 \pi^{2}\left(-\eta^{2}\right)} \nabla_{\mu} \eta^{2} \nabla^{\mu} \eta^{2} .
$$


Being constant, it can be computed at $\theta=\pi / 2, \psi=0$. After a somewhat long but straightforward calculation we find

$$
\begin{aligned}
T_{H} & =\frac{r_{H}}{4 \pi m \cosh \alpha}\left(2 r_{H}^{2}+l_{1}^{2}+l_{2}^{2}+l_{3}^{2}-\frac{l_{1}^{2} l_{2}^{2} l_{3}^{2}}{r_{H}^{4}}\right) \\
& =\frac{1}{4 \pi r_{H} m \cosh \alpha}\left(r_{H}^{2}-r_{1}^{2}\right)\left(r_{H}^{2}-r_{2}^{2}\right),
\end{aligned}
$$

where $r_{H}^{2}, r_{1}^{2}, r_{2}^{2}$ are the three roots of eq. (5). The second line in (8) can be proved by multiplying (5) by $\left(r^{2}-r_{H}^{2}\right)^{-1}$ and taking the limit $r^{2} \rightarrow r_{H}^{2}$. The mass, entropy and angular momenta are given by

$$
\begin{aligned}
M_{\mathrm{ADM}} & =\frac{V_{3} V\left(\Omega_{5}\right)}{4 \pi G_{N}} 2 m\left(\frac{5}{4}+\sinh ^{2} \alpha\right), \quad V\left(\Omega_{5}\right)=\pi^{3}, \\
S & =\frac{V_{3} V\left(\Omega_{5}\right)}{4 G_{N}} 2 m r_{H} \cosh \alpha, \\
J_{i} & =\frac{V_{3} V\left(\Omega_{5}\right)}{4 \pi G_{N}} m l_{i} \cosh \alpha, \quad i=1,2,3
\end{aligned}
$$

where $V_{3}$ is the volume of the 3-brane.

\section{$3 \quad$ WKB method}

Glueball masses in the models of $[2,5]$ have been calculated in the WKB approximation in [14]. By extending the approach of [14], we develop in this section a simple formalism that will be useful to calculate the different mass spectra (including Kaluza-Klein modes) in the present case of three angular momenta.

We are interested in differential equations of the form

$$
\partial_{u}\left(f(u) \partial_{u} \phi\right)+\left(M^{2} h(u)+p(u)\right) \phi=0,
$$

where $M$ represents a mass parameter, and $f(u), h(u)$ and $p(u)$ are three arbitrary functions that are independent of $M$ and have the following behavior. There is a point $u_{H}$, where

$f \approx f_{1}\left(u-u_{H}\right)^{s_{1}}, \quad h \approx h_{1}\left(u-u_{H}\right)^{s_{2}}, \quad p \approx p_{1}\left(u-u_{H}\right)^{s_{3}}, \quad$ as $\quad u \rightarrow u_{H}$,

for some constants $s_{1}, s_{2}, s_{3}, f_{1}, h_{1}$ and $p_{1}$. Similarly, we assume that

$$
f \approx f_{2} u^{r_{1}}, \quad h \approx h_{2} u^{r_{2}}, \quad p \approx p_{2} u^{r_{3}}, \quad \text { as } u \rightarrow \infty,
$$

for some other constants $r_{1}, r_{2}, r_{3}, f_{2}, h_{2}$ and $p_{2}$. For large masses $M$, one may apply WKB methods to obtain the approximate spectrum and 
expressions for $\phi$. In order to apply standard formulae from the WKBapproximation theory we cast (12) into the form of a Schrödinger equation

$$
\partial_{y}^{2} \psi+V(y) \psi=0 \text {. }
$$

The necessary transformation that brings (12) into the form (15) is

$$
e^{y}=u-u_{H}, \quad \phi=e^{\frac{y}{2}} f^{-\frac{1}{2}} \psi,
$$

with the potential given by

$$
\begin{aligned}
& V(y)=M^{2} \frac{h_{0}}{f_{0}}-\frac{1}{2} \frac{f_{0}^{\prime \prime}}{f_{0}}+\frac{1}{4} \frac{f_{0}^{\prime 2}}{f_{0}^{2}}+\frac{p_{0}}{f_{0}}, \\
& f_{0} \equiv e^{-y} f, \quad h_{0} \equiv e^{y} h, \quad p_{0}=e^{y} p .
\end{aligned}
$$

From the asymptotic expressions (13) and (14) one finds that

$$
V(y) \approx \frac{h_{1}}{f_{1}} M^{2} e^{\left(s_{2}-s_{1}+2\right) y}+\frac{p_{1}}{f_{1}} e^{\left(s_{3}-s_{1}+2\right) y}-\frac{1}{4}\left(s_{1}-1\right)^{2}, \quad \text { for } \quad y \ll 0,
$$

and

$$
V(y) \approx \frac{h_{2}}{f_{2}} M^{2} e^{-\left(r_{1}-r_{2}-2\right) y}+\frac{p_{2}}{f_{2}} e^{-\left(r_{1}-r_{3}-2\right) y}-\frac{1}{4}\left(r_{1}-1\right)^{2}, \quad \text { for } \quad y \gg 0 .
$$

Consistency requires that $s_{2}-s_{1}+2$ and $r_{1}-r_{2}-2$ are strictly positive numbers, whereas $s_{3}-s_{1}+2$ and $r_{1}-r_{3}-2$ can be either positive or zero (see also below). From these expressions we see that there are two turning points $y_{1}$ and $y_{2}$, given by solving $V\left(y_{1}\right)=0$ in (18) and $V\left(y_{2}\right)=0$ in (19). To the order of approximation that we will be interested for the computation of $M$, we have

$$
y_{1}=-\frac{2}{\alpha_{1}} \ln \left(\frac{2 \sqrt{\frac{h_{1}}{f_{1}}}}{\alpha_{2}} M\right), \quad y_{2}=\frac{2}{\beta_{1}} \ln \left(\frac{2 \sqrt{\frac{h_{2}}{f_{2}}}}{\beta_{2}} M\right)
$$

where

$$
\alpha_{1}=s_{2}-s_{1}+2, \quad \beta_{1}=r_{1}-r_{2}-2,
$$

and

$$
\begin{aligned}
& \alpha_{2}=\left|s_{1}-1\right| \quad \text { or } \quad \alpha_{2}=\sqrt{\left(s_{1}-1\right)^{2}-4 \frac{p_{1}}{f_{1}}}\left(\text { if } s_{3}-s_{1}+2=0\right) \\
& \beta_{2}=\left|r_{1}-1\right| \quad \text { or } \quad \beta_{2}=\sqrt{\left(r_{1}-1\right)^{2}-4 \frac{p_{2}}{f_{2}}}\left(\text { if } r_{1}-r_{3}-2=0\right) .(22)
\end{aligned}
$$

Then the mass spectrum is computed using the standard WKB formula

$$
\left(m-\frac{1}{2}\right) \pi=\int_{y_{1}}^{y_{2}} d y \sqrt{V(y)}, \quad m \geq 1 .
$$


One may expand the right-hand side as a power series in $\frac{1}{M}$. The leading term is $\mathcal{O}(M)$ and is obtained by keeping, in the expression for $V(y)$ in (17), only the first term, and integrating $y$ from $-\infty$ to $+\infty$. One obtains

$$
\left(m-\frac{1}{2}\right) \pi=M \int_{-\infty}^{\infty} d y \sqrt{\frac{h_{0}}{f_{0}}}=M \int_{u_{H}}^{\infty} d u \sqrt{\frac{h}{f}} \equiv M \xi,
$$

where the last equality defines the constant $\xi$ with scale dimension of length. The first correction of order $\mathcal{O}\left(M^{0}\right)$ has a contribution from the term

$$
-\sqrt{\frac{h_{1}}{f_{1}}} M \int_{-\infty}^{y_{1}} d y e^{\frac{1}{2} \alpha_{1} y}-\sqrt{\frac{h_{2}}{f_{2}}} M \int_{y_{2}}^{\infty} d y e^{-\frac{1}{2} \beta_{1} y}=-\frac{a_{2}}{a_{1}}-\frac{b_{2}}{b_{1}}
$$

representing the correction that accounts for the extension of the limits of integration from $y_{1,2}$ to $\mp \infty$. There is another subleading contribution from around the turning points

$$
\begin{gathered}
\int_{y_{1}}^{\infty} d y\left(\sqrt{\frac{h_{1}}{f_{1}} M^{2} e^{-\beta_{1} y}-\frac{\beta_{2}^{2}}{4}}-\sqrt{\frac{h_{1}}{f_{1}}} M e^{-\frac{1}{2} \beta_{1} y}\right) \\
+\int_{-\infty}^{y_{2}} d y\left(\sqrt{\frac{h_{2}}{f_{2}} M^{2} e^{\alpha_{1} y}-\frac{\alpha_{2}^{2}}{4}}-\sqrt{\frac{h_{2}}{f_{2}}} M e^{\frac{1}{2} \alpha_{1} y}\right)=\frac{1}{2}(2-\pi)\left(\frac{\alpha_{2}}{\alpha_{1}}+\frac{\beta_{2}}{\beta_{1}}\right) .
\end{gathered}
$$

Combining everything we find that

$$
M^{2}=\frac{\pi^{2}}{\xi^{2}} m\left(m-1+\frac{\alpha_{2}}{\alpha_{1}}+\frac{\beta_{2}}{\beta_{1}}\right)+\mathcal{O}\left(m^{0}\right), \quad m \geq 1 .
$$

The validity of the WKB approximation requires that $\alpha_{2} / \alpha_{1}+\beta_{2} / \beta_{1} \ll m$ and that the turning points, as computed using (20), be large in magnitude. This gives the conditions

$$
m\left(m-1+\frac{\alpha_{2}}{\alpha_{1}}+\frac{\beta_{2}}{\beta_{1}}\right) \gg \frac{f_{1} \alpha_{2}^{2}}{h_{1}} \xi^{2} u_{H}^{-\alpha_{1}} \text { and } \frac{f_{2} \beta_{2}^{2}}{h_{2}} \xi^{2} u_{H}^{\beta_{1}} .
$$

\section{Glueball masses in $\mathrm{QCD}_{3}$}

The three-parameter $\mathrm{QCD}_{3}$ model is obtained from the rotating D3-brane metric (1) in the following way. We first go to Euclidean space by letting $t \rightarrow-i \tau$ and $l_{i} \rightarrow i l_{i}, i=1,2,3$. Then we take the "field-theory" limit $[1,5]$

$$
U=\frac{r}{\alpha^{\prime}}, \quad U_{0}^{4}=\frac{2 m}{\alpha^{\prime 4}}, \quad a_{i}=\frac{l_{i}}{\alpha^{\prime}}, \quad \alpha^{\prime} \rightarrow 0 .
$$


The rotating D3-brane metric in this limit takes the form

$$
\begin{aligned}
d s_{\mathrm{IIB}}^{2} & =\alpha^{\prime} \Delta^{1 / 2}\left[\frac{U^{2}}{R^{2}}\left[\left(1-\frac{U_{0}^{4}}{U^{4} \Delta}\right) d \tau^{2}+d x_{1}^{2}+d x_{2}^{2}+d x_{3}^{2}\right]\right. \\
& +\frac{R^{2} d U^{2}}{U^{2}\left[\prod_{i=1}^{3}\left(1-\frac{a_{i}^{2}}{U^{2}}\right)-\frac{U_{0}^{4}}{U^{4}}\right]}+\frac{R^{2}}{\Delta}\left(\Delta_{1} d \theta^{2}+\Delta_{2} \cos ^{2} \theta d \psi^{2}\right. \\
& +2 \frac{a_{2}^{2}-a_{3}^{2}}{U^{2}} \cos \theta \sin \theta \cos \psi \sin \psi d \theta d \psi+\left(1-\frac{a_{1}^{2}}{U^{2}}\right) \sin ^{2} \theta d \varphi_{1}^{2} \\
& \left.+\left(1-\frac{a_{2}^{2}}{U^{2}}\right) \cos ^{2} \theta \sin ^{2} \psi d \varphi_{2}^{2}+\left(1-\frac{a_{3}^{2}}{U^{2}}\right) \cos ^{2} \theta \cos ^{2} \psi d \varphi_{3}^{2}\right) \\
& \left.-\frac{2 U_{0}^{2}}{U^{2} \Delta} d \tau\left(a_{1} \sin ^{2} \theta d \varphi_{1}+a_{2} \cos ^{2} \theta \sin ^{2} \psi d \varphi_{2}+a_{3} \cos ^{2} \theta \cos ^{2} \psi d \varphi_{3}\right)\right],
\end{aligned}
$$

where $R^{2}=\sqrt{4 \pi g_{s} N}=$ fixed, and

$$
\begin{aligned}
\Delta & =1-\frac{a_{1}^{2}}{U^{2}} \cos ^{2} \theta-\frac{a_{2}^{2}}{U^{2}}\left(\sin ^{2} \theta \sin ^{2} \psi+\cos ^{2} \psi\right)-\frac{a_{3}^{2}}{U^{2}}\left(\sin ^{2} \theta \cos ^{2} \psi\right. \\
& \left.+\sin ^{2} \psi\right)+\frac{a_{2}^{2} a_{3}^{2}}{U^{4}} \sin ^{2} \theta+\frac{a_{1}^{2} a_{3}^{2}}{U^{4}} \cos ^{2} \theta \sin ^{2} \psi+\frac{a_{1}^{2} a_{2}^{2}}{U^{4}} \cos ^{2} \theta \cos ^{2} \psi, \\
\Delta_{1} & =1-\frac{a_{1}^{2}}{U^{2}} \cos ^{2} \theta-\frac{a_{2}^{2}}{U^{2}} \sin ^{2} \theta \sin ^{2} \psi-\frac{a_{3}^{2}}{U^{2}} \sin ^{2} \theta \cos ^{2} \psi, \\
\Delta_{2} & =1-\frac{a_{2}^{2}}{U^{2}} \cos ^{2} \psi-\frac{a_{3}^{2}}{U^{2}} \sin ^{2} \psi .
\end{aligned}
$$

For large $U$ the space becomes asymptotically $A d S_{5} \times S^{5}$, each factor having radius $R$ (in string units). In the limit (29) the Hawking temperature is given by

$$
\begin{aligned}
T_{H} & =\frac{U_{H}}{2 \pi R^{2} U_{0}^{2}}\left(2 U_{H}^{2}-a_{1}^{2}-a_{2}^{2}-a_{3}^{2}+\frac{a_{1}^{2} a_{2}^{2} a_{3}^{2}}{U_{H}^{4}}\right) \\
& =\frac{1}{2 \pi R^{2} U_{0}^{2} U_{H}}\left(U_{H}^{2}-U_{1}^{2}\right)\left(U_{H}^{2}-U_{2}^{2}\right),
\end{aligned}
$$

$U_{H}^{2}$ being the largest real root of $f(U)=0$ :

$$
f=\prod_{i=1}^{3}\left(U^{2}-a_{i}^{2}\right)-U_{0}^{4} U^{2}=\left(U^{2}-U_{H}^{2}\right)\left(U^{2}-U_{1}^{2}\right)\left(U^{2}-U_{2}^{2}\right),
$$

and $U_{1}^{2}, U_{2}^{2}$ the two other roots. The Yang-Mills coupling of the $(2+1)$ dimensional field theory is given by

$$
g_{\mathrm{YM}_{3}}^{2}=g_{\mathrm{YM}_{4}}^{2} T_{H}, \quad g_{\mathrm{YM}_{4}}^{2}=2 \pi g_{s} .
$$

The 't Hooft coupling $\lambda$ is defined by $\lambda \equiv g_{\mathrm{YM}_{3}}^{2} N /(2 \pi)=T_{H} R^{4} /(4 \pi)$. 
In what follows, we will make use of the following formulas

$$
\sqrt{G}=R^{2} \Delta^{1 / 2} U^{3} \cos ^{3} \theta \sin \theta \sin \psi \cos \psi,
$$

and

$$
\begin{aligned}
& G^{\psi \psi}=\frac{\Delta_{1}}{R^{2} \cos ^{2} \theta \Delta^{1 / 2}}, \quad G^{\theta \psi}=-\frac{a_{2}^{2}-a_{3}^{2}}{R^{2} \Delta^{1 / 2} U^{2}} \tan \theta \cos \psi \sin \psi \\
& G^{\theta \theta}=\frac{\Delta_{2}}{R^{2} \Delta^{1 / 2}}, \quad G^{\tau \tau}=\frac{R^{2}}{U^{2} \Delta^{1 / 2}} \frac{\prod_{i=1}^{3}\left(1-\frac{a_{i}^{2}}{U^{2}}\right)}{\prod_{i=1}^{3}\left(1-\frac{a_{i}^{2}}{U^{2}}\right)-\frac{U_{0}^{4}}{U^{4}}} .
\end{aligned}
$$

\section{$4.10^{++}$glueballs}

Masses for $0^{++}$glueballs are determined from the differential equation

$$
\frac{1}{\sqrt{G}} \partial_{\mu} e^{-2 \Phi} \sqrt{G} G^{\mu \nu} \partial_{\nu} \Psi=0
$$

We look for solutions of the form $\Psi=\phi(u) e^{i k \cdot x}$ and identify the glueball mass square with $M^{2}=-k^{2}$ [2]. After changing variable as $u=U^{2}$ (and $u_{0}=U_{0}^{2}$ etc.), we find an equation of the form (12) with

$$
f=\prod_{i=1}^{3}\left(u-a_{i}^{2}\right)-u_{0}^{2} u, \quad h=\frac{R^{4}}{4}, \quad p=0 .
$$

The constant $u_{H}$ is found by solving the cubic equation $f=0$, which can be written as

$$
\begin{aligned}
& u_{H}^{3}-\vec{a}^{2} u_{H}^{2}+\left(\vec{b}^{2}-u_{0}^{2}\right) u_{H}-c=0, \\
& c=a_{1}^{2} a_{2}^{2} a_{3}^{2}, \quad \vec{a}=\left(a_{1}, a_{2}, a_{3}\right), \quad \vec{b}=\left(a_{2} a_{3}, a_{1} a_{3}, a_{1} a_{2}\right) .
\end{aligned}
$$

For the various constants necessary for the application of the WKB method of Sect. 3, we find

$$
\begin{aligned}
& s_{1}=1, \quad s_{2}=0, \quad r_{1}=3, \quad r_{2}=0, \\
& \alpha_{1}=1, \quad \alpha_{2}=0, \quad \beta_{1}=1, \quad \beta_{2}=2 \text {. }
\end{aligned}
$$

Therefore (27) gives

$$
\begin{aligned}
& M^{2}=\frac{\pi^{2}}{\xi^{2}} m(m+1)+\mathcal{O}\left(m^{0}\right), \quad m \geq 1, \\
& \xi=\frac{R^{2}}{2} \int_{u_{H}}^{\infty} d u\left(u^{3}-\vec{a}^{2} u^{2}+\left(\vec{b}^{2}-u_{0}^{2}\right) u-c\right)^{-1 / 2} .
\end{aligned}
$$

The integral in (41) can be performed explicitly. Let $u_{H}, u_{1}$ and $u_{2}$ be the roots of $f=0$. One obtains

$\xi=\frac{R^{2} K(k)}{\left[\left(u_{H}-u_{1}\right)\left(u_{H}-u_{2}\right)\right]^{1 / 4}}, k=\frac{1}{\sqrt{2}}\left(1-\frac{2 u_{H}-u_{1}-u_{2}}{2\left[\left(u_{H}-u_{1}\right)\left(u_{H}-u_{2}\right)\right]^{1 / 2}}\right)^{1 / 2}$, 
where $K(k)$ is the complete elliptic integral of the first kind, i.e. $K(k)=$ $\int_{0}^{\pi / 2} \frac{d \theta}{\sqrt{1-k^{2} \sin ^{2} \theta}}$. We may distinguish two cases, according to whether $u_{1}$ and $u_{2}$ are real or complex. In the former case the above result can also be written as $\left(u_{H}>u_{1}>u_{2}\right)$

$$
\xi=\frac{R^{2} K\left(k_{0}\right)}{\sqrt{u_{H}-u_{2}}}, \quad k_{0}=\sqrt{\frac{u_{1}-u_{2}}{u_{H}-u_{2}}} .
$$

The validity of the WKB approximation requires that the conditions (28) be satisfied. These imply

$$
m(m+1) \gg\left(1-\frac{u_{1}}{u_{H}}\right)^{-1 / 2}\left(1-\frac{u_{2}}{u_{H}}\right)^{-1 / 2} K^{2}(k) .
$$

In the case of three real roots $u_{H}>u_{1}>u_{2}$, and $u_{1}$ not too close to $u_{H}$, $k$ is always less than 1 , so that $K(k)$ is of order 1 , and (44) implies that the WKB approximation can be applied. It breaks down when $u_{H} \simeq u_{1}$. For the case of 1 real and 2 complex roots, this happens if $\operatorname{Im} u_{1} \simeq 0$ with $u_{H} \simeq \operatorname{Re} u_{1}$ (or with $u_{H}<\operatorname{Re} u_{1}$, in which case $k \simeq 1$ ).

If $c=a_{1} a_{2} a_{3}=0$ (say, $a_{3}=0$ ), there are then three real roots - one of them being $u_{3}=0-$, since $\left(\vec{a}^{2}\right)^{2}-4 \vec{b}^{2}+4 u_{0}^{2} \geq 0$. The situation is similar to the one-angular momentum case discussed in $[5,7,14]$. The case $a_{1}=a_{2}$, $u_{0}=0$ is special. This gives a double root with $u_{1}=u_{2}=a_{1}^{2}$, so that $s_{1}=2$, $r_{1}=3, s_{2}=r_{2}=0$ and $s_{2}-s_{1}+2=0$ and hence the WKB method breaks down (cf. eq. (19)). breaks down. The WKB method breaks down also in the case $a_{1}=a_{2}=a_{3} \gg u_{0}$ (see also below).

The formula for the mass spectrum (41) for $0^{++}$glueballs and its resonances implies an important prediction for the ratio between masses of two arbitrary resonances: it is independent of $a_{i}$ (up to corrections of order $\left.1 / m^{2}\right)$, depending only on the radial quantum numbers

$$
\frac{M_{m}^{2}}{M_{m^{\prime}}^{2}} \cong \frac{m(m+1)}{m^{\prime}\left(m^{\prime}+1\right)} .
$$

This was observed in [14] for the case of $\mathrm{QCD}_{4}$ with one angular-momentum. It implies that glueball masses with $m \gg 1$ vary only slightly in the whole parameter space $a_{1}, a_{2}, a_{3}$.

\subsection{Kaluza-Klein modes with $\tau$ dependence}

For particles with $U(1)$ charge associated with the circle parametrized by $\tau$ one can check that there exist simple (angular-independent) solutions of the form

$$
\Psi=\phi(U) e^{i k \cdot x} e^{2 \pi i n T_{H} \tau} .
$$


Substituting this into eq. (37) we obtain

$$
\begin{aligned}
& \partial_{U} U^{5}\left(\prod_{i=1}^{3}\left(1-\frac{a_{i}^{2}}{U^{2}}\right)-\frac{U_{0}^{4}}{U^{4}}\right) \partial_{U} \phi \\
& +R^{2}\left(U M^{2} R^{2}-4 \pi^{2} n^{2} T_{H}^{2} U^{3} \Delta^{1 / 2} G^{\tau \tau}\right) \phi=0
\end{aligned}
$$

where $G^{\tau \tau}$ is given in eq. (36). Introducing a new radial coordinate $u=U^{2}$ we obtain eq. (12) with $M^{2}$ replaced by $M^{2}-4 \pi^{2} n^{2} T_{H}^{2}$ and

$$
\begin{aligned}
& f=u^{3}-\vec{a}^{2} u^{2}+\left(\vec{b}^{2}-u_{0}^{2}\right) u-c, \quad h=\frac{R^{4}}{4}, \\
& p=-\frac{R^{4} \pi^{2} n^{2} T_{H}^{2} u_{0}^{2} u}{u^{3}-\vec{a}^{2} u^{2}+\left(\vec{b}^{2}-u_{0}^{2}\right) u-c} .
\end{aligned}
$$

Note that this equation is invariant under cyclic permutations of $\left(a_{1}, a_{2}, a_{3}\right)$. We find

$$
\begin{array}{ll}
s_{1}=1, & s_{2}=0, \quad s_{3}=-1, \quad r_{1}=3, \quad r_{2}=0, \quad r_{3}=-2, \\
\alpha_{1}=1, & \alpha_{2}=\frac{2 R^{2} \pi n T_{H} u_{0} \sqrt{u_{H}}}{\left(u_{H}-u_{1}\right)\left(u_{H}-u_{2}\right)}, \quad \beta_{1}=1, \quad \beta_{2}=2 .
\end{array}
$$

Using (32) we see that $\alpha_{2}=n$. Then (27) (with $M^{2} \rightarrow M^{2}-4 \pi^{2} n^{2} T_{H}^{2}$ ) gives the formula

$$
M^{2}=4 \pi^{2} n^{2} T_{H}^{2}+\frac{\pi^{2}}{\xi^{2}} m(m+1+n)+\mathcal{O}\left(m^{0}\right), \quad m \geq 1,
$$

where $\xi$ is given by (42). Using (28) we obtain that WKB is valid when

$$
\begin{aligned}
& m(m+1+n) \gg\left(u_{H}-u_{1}\right)^{1 / 2}\left(u_{H}-u_{2}\right)^{1 / 2} \frac{n^{2} K^{2}(k)}{u_{H} R^{4}} \text { and } \\
& \frac{u_{H} K^{2}(k)}{\left(u_{H}-u_{1}\right)^{1 / 2}\left(u_{H}-u_{2}\right)^{1 / 2}} .
\end{aligned}
$$

In order to obtain $\mathrm{QCD}_{3}$ through a dimensional reduction of $\mathrm{QCD}_{4}$, it is necessary that the mass scale for these Kaluza-Klein states be much larger than that for the glueball masses. From eq. (50) one sees that this requires the condition

$$
T_{H} \gg \frac{1}{\xi}
$$

Using (32) and (42) this becomes

$$
K(k) \frac{u_{H}}{u_{0}}\left[\left(1-\frac{u_{1}}{u_{H}}\right)\left(1-\frac{u_{2}}{u_{H}}\right)\right]^{3 / 4} \gg 1,
$$


where $k$ is given by (42). $K(k)$ cannot be too large, otherwise the WKB approximation breaks down (see (51)). Therefore eq. (53) implies that in order to decouple the $\tau$ Kaluza-Klein modes one needs that $u_{H} \gg u_{0}$. With no loss of generality we can assume that $a_{1} \geq a_{2} \geq a_{3}$. It is clear from the equation for the horizon that one has $u_{H} \gg u_{0}$ if and only if $u_{H} \simeq a_{1}^{2}$. Thus the region where these Kaluza-Klein particles decouple is $a_{1}^{2} \gg u_{0}$. To ensure at the same time the validity of the WKB approximation (51) one needs that $u_{H}$ be not too close to $u_{1}$, which for $u_{H} \gg u_{0}$ amounts to saying that $a_{1}^{2}$ should not be too close to $a_{2}^{2}$.

\subsection{Kaluza-Klein modes of $S^{5}$}

The isometry group of the metric with $a_{1}=a_{2}=a_{3}=0$ contains a factor $S O(6)$. We will investigate the problem of decoupling for the $l=1$ KaluzaKlein modes - corresponding to the 6 representation of $S O(6)$-, as modes with larger value for $l$ are expected to be heavier. The three angular momenta break the symmetry down to the Cartan subgroup $S O(2) \times S O(2) \times$ $S O(2)$. With respect to the Cartan subgroup, the representation 6 decomposes into three doublets of $S O(2)$, i.e. $\mathbf{6} \rightarrow(\mathbf{2}, \mathbf{1}, \mathbf{1}) \oplus(\mathbf{1}, \mathbf{2}, \mathbf{1}) \oplus(\mathbf{1}, \mathbf{1}, \mathbf{2})$. These give rise to three equations, which must be related by cyclic permutations of $\left(a_{1}, a_{2}, a_{3}\right)$. For the first doublet, we make the ansatz

$$
\Psi=\phi(U) e^{i k \cdot x} \sin \theta\left(\begin{array}{c}
\cos \varphi_{1} \\
\sin \varphi_{1}
\end{array}\right),
$$

and insert it into the Laplace equation (37). One then obtains for $\phi(U)$ the differential equation

$$
\begin{aligned}
& \partial_{U} U^{5}\left(\prod_{i=1}^{3}\left(1-\frac{a_{i}^{2}}{U^{2}}\right)-\frac{U_{0}^{4}}{U^{4}}\right) \partial_{U} \phi+M^{2} R^{4} U \phi \\
& +\left(U^{3}\left(\Delta_{2}\left(\cot ^{2} \theta-4\right)-R^{2} \Delta^{1 / 2} G^{\varphi_{1} \varphi_{1}}\right)-2\left(a_{2}^{2}-a_{3}^{2}\right) U \cos 2 \psi\right) \phi=0
\end{aligned}
$$

where $M^{2}=-k^{2}$. After changing variable $u=U^{2}$, and a somewhat lengthy computation, we see that all $\theta$ and $\psi$ dependence cancels out, and we obtain an equation of the form (12) with

$$
\begin{aligned}
& f=u^{3}-\vec{a}^{2} u^{2}+\left(\vec{b}^{2}-u_{0}^{2}\right) u-c, \quad h=\frac{R^{4}}{4} \\
& p=\frac{1}{4} \frac{-5 u^{4}+c_{3} u^{3}+c_{2} u^{2}+c_{1} u+c_{0}}{u^{3}-\vec{a}^{2} u^{2}+\left(\vec{b}^{2}-u_{0}^{2}\right) u-c}
\end{aligned}
$$

where

$$
c_{3}=4\left(2 \vec{a}^{2}-a_{1}^{2}\right)=4\left(a_{1}^{2}+2 a_{2}^{2}+2 a_{3}^{2}\right),
$$




$$
\begin{aligned}
c_{2} & =-3\left(\left(\vec{a}^{2}\right)^{2}-a_{1}^{4}+2 b_{1}^{2}\right)+5 U_{0}^{4} \\
& =-6 a_{1}^{2} a_{2}^{2}-3 a_{2}^{4}-6 a_{1}^{2} a_{3}^{2}-12 a_{2}^{2} a_{3}^{2}-3 a_{3}^{4}+5 u_{0}^{2}, \\
c_{1} & =8 c+\left(4 b_{1}^{2}-3 u_{0}^{2}\right)\left(\vec{a}^{2}-a_{1}^{2}\right)+2 a_{1}^{2}\left(\left(\vec{a}^{2}\right)^{2}-2 \vec{b}^{2}-a_{1}^{4}\right) \\
& =2 a_{1}^{2} a_{2}^{4}+8 a_{1}^{2} a_{2}^{2} a_{3}^{2}+4 a_{2}^{4} a_{3}^{2}+2 a_{1}^{2} a_{3}^{4}+4 a_{2}^{2} a_{3}^{4}-3\left(a_{2}^{2}+a_{3}^{2}\right) u_{0}^{2} \\
c_{0} & =-b_{1}^{2}\left(2 \vec{b}^{2}-b_{1}^{2}-u_{0}^{2}\right)=-a_{2}^{2} a_{3}^{2}\left(2 a_{1}^{2} a_{2}^{2}+2 a_{1}^{2} a_{3}^{2}+a_{2}^{2} a_{3}^{2}-u_{0}^{2}\right) .
\end{aligned}
$$

When $a_{2}=a_{3}=0$, the differential equation reduces to eq. (3.15) of [7] (with $\left.u \rightarrow u^{2}\right)$. For $a_{1}=a_{3}=0$, it reduces to eq. (3.16) of [7], which corresponds to the other doublet (this is because interchanging doublets is equivalent to permuting $\left.a_{1}, a_{2}, a_{3}\right)$. For the various constants of Sect. 3 we find

$$
\begin{aligned}
& s_{1}=1, \quad s_{2}=0, \quad s_{3}=-1, \quad r_{1}=3, \quad r_{2}=0, \quad r_{3}=1 \\
& f_{1}=\left(u_{H}-u_{1}\right)\left(u_{H}-u_{2}\right), \quad f_{2}=1, \quad p_{1}=-\frac{a_{1}^{2}\left(u_{H}-a_{2}^{2}\right)^{2}\left(u_{H}-a_{3}^{2}\right)^{2}}{4 u_{H} f_{1}}, \\
& p_{2}=-\frac{5}{4}, \alpha_{1}=1, \quad \alpha_{2}=\frac{a_{1}\left(u_{H}-a_{2}^{2}\right)\left(u_{H}-a_{3}^{2}\right)}{\sqrt{u_{H}} f_{1}}, \quad \beta_{1}=1 \\
& \beta_{2}=3 .
\end{aligned}
$$

Hence, using (28), we find the mass

$$
M^{2}=\frac{\pi^{2}}{\xi^{2}} m\left(m+2+\frac{a_{1}\left(u_{H}-a_{2}^{2}\right)\left(u_{H}-a_{3}^{2}\right)}{\sqrt{u_{H}}\left(u_{H}-u_{1}\right)\left(u_{H}-u_{2}\right)}\right)+\mathcal{O}\left(m^{0}\right), \quad m \geq 1,
$$

where $\xi$ is given by (42).

Let us examine if these states may have a large mass in the same region $a_{1}^{2} \gg u_{0}$ where the Kaluza-Klein modes with $\tau$ dependence decouple. In this limit one has $u_{H} \cong a_{1}^{2}, u_{1} \cong a_{2}^{2}, u_{2} \cong a_{3}^{2}$, and the mass formula (59) takes the form

$$
M^{2} \simeq \frac{\pi^{2}}{\xi^{2}} m(m+3)+\mathcal{O}\left(m^{0}\right), \quad m \geq 1,
$$

where $\xi$ is again given by (42). This shows that for $a_{1}^{2} \gg u_{0}$ the mass of these Kaluza-Klein states is of the same order as the glueball masses (41). More generally, one can show that in every region of the parameter space the Kaluza-Klein masses (59) are of order $M=\mathcal{O}(1 / \xi)$. Indeed, it could only be otherwise in a region where $u_{H} \cong u_{1}$, where the third term of (59) has a potential singularity, but this happens only in the region $a_{1}^{2} \gg u_{0}$, which leads to $(60)$.

For the ansatz

$$
\Psi=\phi(U) e^{i k \cdot x} \cos \theta \sin \psi\left(\begin{array}{c}
\cos \varphi_{2} \\
\sin \varphi_{2}
\end{array}\right)
$$


and for

$$
\Psi=\phi(U) e^{i k \cdot x} \cos \theta \cos \psi\left(\begin{array}{c}
\cos \varphi_{3} \\
\sin \varphi_{3}
\end{array}\right),
$$

corresponding to the other two doublets, we obtain the cyclic permutation in $\left(a_{1}, a_{2}, a_{3}\right)$ of (56), as expected. Note that the constants $c_{0}, \ldots, c_{3}$ in (57) are not invariant under such permutations. The constants of (58) are obtained by cyclic permutation in $\left(a_{1}, a_{2}, a_{3}\right)$ of (58). For completeness we include the corresponding mass spectra. For the Kaluza-Klein doublet (61) it is given by

$$
M^{2}=\frac{\pi^{2}}{\xi^{2}} m\left(m+2+\frac{a_{2}\left(u_{H}-a_{3}^{2}\right)\left(u_{H}-a_{1}^{2}\right)}{\sqrt{u_{H}}\left(u_{H}-u_{1}\right)\left(u_{H}-u_{2}\right)}\right)+\mathcal{O}\left(m^{0}\right), \quad m \geq 1,
$$

and for the Kaluza-Klein doublet (62) it is given by

$$
M^{2}=\frac{\pi^{2}}{\xi^{2}} m\left(m+2+\frac{a_{3}\left(u_{H}-a_{1}^{2}\right)\left(u_{H}-a_{2}^{2}\right)}{\sqrt{u_{H}}\left(u_{H}-u_{1}\right)\left(u_{H}-u_{2}\right)}\right)+\mathcal{O}\left(m^{0}\right), \quad m \geq 1,
$$

where $\xi$ is given by (42) in both mass formulae. In the region $a_{1}^{2} \gg u_{0}$ where the circle Kaluza-Klein states decouple, eqs. (63) and (64) take the form

$$
M^{2} \simeq \frac{\pi^{2}}{\xi^{2}} m(m+2)+\mathcal{O}\left(m^{0}\right), \quad m \geq 1 .
$$

These are essentially the same mass formulae as in the case of the $0^{++}$ glueballs, so that the corresponding mass scales are the same.

Finally, we note that the conditions for the validity of the WKB approximation in the case of the $S^{5}$ Kaluza-Klein modes are roughly the same as the corresponding ones for the $0^{++}$glueballs we have already discussed. In all cases the WKB approximation can be applied everywhere, except in the region $a_{1}^{2}=a_{2}^{2} \gg u_{0}$.

\section{Conclusions}

In this paper we have considered non-supersymmetric QCD models in $2+1$ dimensions based on an asymptotically $A d S_{5} \times S^{5}$ static geometry constructed from the rotating $\mathrm{D} 3$-brane with maximum number of rotation parameters. Within the WKB approximation, closed analytic formulas have been obtained for the mass spectra of $0^{++}$scalar glueballs and of states corresponding to excitations in the internal parts (circle and sphere) of the space. The various mass spectra were found to depend, for every model and to the first two leading orders in the WKB approximation, in a universal manner on the rotation parameters: modulo slight fluctuations (such as the one 
produced by the third term in (59)), only two mass scales appear, denoted $1 / \xi$ and $T_{H}$, the latter characterizing the masses of Kaluza-Klein particles associated with the $\tau$ direction, the former dictating the masses of all other scalar modes with vanishing charge in the $\tau$ direction. This is a bit surprising, and it implies that, despite the large number of parameters, the sphere Kaluza-Klein modes - unlike the ones corresponding to the circle - do not decouple in any region of parameter space. The sphere Kaluza-Klein states have no analogue at the weakly coupled finite- $N$ pure $S U(N)$ Yang-Mills theories. Masses computed in the supergravity approximation can in principle get important corrections upon extrapolating from the strong-coupling regime $\lambda \gg 1$ to the weak-coupling regime $\lambda \ll 1$. Comparison with lattice results $[4,7]$ suggests that singlet particles should only be slightly changed in the extrapolation process, whereas non-singlet particles should get large corrections.

The extremal solution with $u_{0}=0$ saturates the Bogomol'nyi bound and has unbroken supersymmetries. The near-supersymmetric case $a_{i}^{2} \gg u_{0}$ is precisely the interesting case where the radius of the extra brane direction shrinks to zero, so that the associated Kaluza-Klein particles decouple and the system becomes effectively $(2+1)$-dimensional. It would be very interesting to establish what are the states whose masses are protected by supersymmetry in the rotating system with $u_{0}=0$. This might explain why glueball masses have values close to the values obtained by lattice calculations $[7,15]$.

\section{Acknowledgements}

We have benefited from discussions with C. Csáki and J. Terning on closely related matters. J.R. would like to thank Fundación Antorchas for financial support (project A-13681/1). 


\section{References}

[1] J. M. Maldacena, Adv. Theor. Math. Phys. 2 (1998) 231, hep-th/9711200.

[2] E. Witten, Adv. Theor. Math. Phys. 2 (1998) 505, hep-th/9803131.

[3] D. J. Gross and H. Ooguri, Phys. Rev. D58 (1998) 106002, hep-th/9805129.

[4] C. Csáki, H. Ooguri, Y. Oz and J. Terning, Glueball mass spectrum from supergravity, JHEP 9901 (1999) 017, hep-th/9806021.

[5] J.G. Russo, New compactifications of supergravities and large $N Q C D$, Nucl. Phys. B543 (1999) 183, hep-th/9808117.

[6] A. Hashimoto and Y. Oz, Aspects of QCD dynamics from string theory, hep-th/9809106.

[7] C. Csáki, Y. Oz, J.G. Russo and J. Terning, Large- $N$ QCD from rotating branes, Phys.. Rev. D59 (1999) 065008, hep-th/9810186.

[8] H. Ooguri, H. Robins and J. Tannenhauser, Phys. Lett. B437 (1998) 77, hep-th/9806171.

[9] P. Kraus, F. Larsen and S.P. Trivedi, The Coulomb branch of gauge theory from rotating branes, JHEP 9903 (1999) 003, hep-th/9811120.

[10] M. Cvetic and D. Youm, Nucl. Phys. B477 (1996) 449, hep-th/9605051.

[11] S. S. Gubser, Thermodynamics of spinning D3-branes, hep-th/9810225.

[12] R.G. Cai and K.S Soh, Critical behavior in the rotating D-branes, hep-th/9812121.

[13] K. Sfetsos, Branes for Higgs phases and exact conformal field theories, JHEP 9901 (1999) 015, hep-th/9811167.

[14] J.A. Minahan, Glueball mass spectra and other issues for supergravity duals of QCD models, JHEP 9901 (1999) 020, hep-th/9811156.

[15] M.J. Teper, Phys. Rev. D59 (1999) 014512, hep-lat/9804008. 